\title{
Emotional valence influences the neural correlates associated with remembering and knowing
}

\author{
Katherine R. Mickley and Elizabeth A. Kensinger \\ Boston College, Chestnut Hill, Massachusetts \\ and Athinoula A. Martinos Center for Biomedical Imaging, Charlestown, Massachusetts
}

\begin{abstract}
In the present study, we examined whether emotional valence modulates the neural processes that are engaged during the encoding of information that is later vividly remembered versus that which is only known to be familiar. Participants underwent an fMRI scan while viewing positive, negative, and neutral stimuli. Later, recognized items were labeled as either remembered or known. Negative items that were later vividly remembered recruited temporo-occipital regions associated with sensory processing more than did positive or neutral items that were vividly remembered. The encoding of positive information later known recruited the cingulate gyrus and bilateral frontal and parietal areas - regions associated with episodic and semantic retrieval and self-referential processing - more than did the encoding of negative or neutral items that were later known. These results suggest that memories for negative items may be vividly recollected due to increased sensory processing during encoding, whereas enhanced gist-based processing of positive information may lead to increased feelings of familiarity.
\end{abstract}

Information can be recognized with varying amounts of detail. Some items are recollected with crisp visual detail or with vivid contextual details that make us sure we have seen the items before. Other items leave behind only a haze of familiarity: We know that we have seen the items before, but we can't quite pin down why we know this. When participants recognize an item as a studied one, they could be using either recollection or familiarity to support their decisions. A common way to distinguish the cognitive processes is to ask participants whether they are able to vividly "remember" the item or whether they simply "know" that it was presented because it is familiar to them. A "remember" response indicates recollection of the episodic details, whereas a "know" response reflects item familiarity in the absence of recollection.

Although it has been debated to what extent these different forms of recognition are supported by distinct processes (see Jacoby, 1991; Mandler, 1980; Slotnick \& Dodson, 2005; Wais, Wixted, Hopkins, \& Squire, 2006; Yonelinas, 2002), there is increasing evidence to suggest that vividly remembering information is supported by different processes than those used when simply knowing that something is familiar. In particular, items that are later vividly remembered are associated with activity in the hippocampus and in the posterior parahippocampus, whereas items that are later only known to be familiar tend to be associated with activity in the rhinal cortex (see Davachi \& Wagner, 2002; Dobbins, Kroll, \& Yonelinas, 2004; Kensinger, Clarke, \& Corkin, 2003; Ranganath et al., 2003). Prefrontal contributions during encoding also may influence the likelihood that an item is later remembered vividly (Davidson \& Glisky, 2002; Kensinger et al., 2003; Knowlton \& Squire, 1995; Manns, Hopkins, Reed, Kitchener, \& Squire, 2003); although prefrontal regions can support both vivid remembering and feelings of familiarity, subregions of the ventrolateral prefrontal cortex may be particularly important for vivid recollections (Ranganath et al., 2003).

It has been less clear whether the emotional valence of information has an influence on these general dissociations between the neural systems associated with subsequent remembering and knowing. The few studies that have focused on the neural processes supporting recollection of emotional information have suggested that the amygdala plays a critical role, both at encoding (Adolphs, Tranel, \& Buchanan, 2005; Kensinger \& Schacter, 2005) and at retrieval (Sharot, Delgado, \& Phelps, 2004). By contrast, the amygdala plays no role in the recollection of neutral information (see Phelps, 2006). Although this finding might suggest that distinct processes support the vivid remembering of emotional as compared with nonemotional items, it does appear that the amygdala exerts many of its effects through interactions with the hippocampus proper (Packard, Cahill, \& McGaugh, 1994) — a key player in the recollection of neutral information (Kensinger \& Corkin, 2004; Richardson, Strange, \& Dolan, 2004). Moreover, medial temporal-lobe dissociations (i.e., the hippocampus proper corresponding with later memory for detail and the rhinal cortex corresponding with later item familiarity) do 
sometimes hold for emotional items as well as for nonemotional ones (e.g., Kensinger \& Schacter, 2006).

Although these studies suggest overlap along with points of divergence in the processes contributing to the recollection of emotional and nonemotional information, it is less clear how the valence of information (whether it is positive or negative) affects these processes. It has been proposed that negative and positive items may be processed in fundamentally different ways, with negative emotion evoking detail-oriented and analytical processing, but positive emotion eliciting a schematic, heuristic type of processing (for reviews, see Bless, Schwarz, \& Wieland, 1996; Fredrickson, 2004). If negative and positive items are processed differently during encoding, then different neural processes may correspond with their later recognition.

There is some evidence that distinct prefrontal processes support the encoding of pleasant and unpleasant information (Dolcos, LaBar, \& Cabeza, 2004). However, there does not appear to be divergence throughout the mnemonic network supporting the successful encoding of positive and negative information. For example, a study by Kensinger and Schacter (2006) revealed similar patterns of medial temporal-lobe activity corresponding with subsequent memory for positive and negative items. Because prior studies have taken a region-of-interest (ROI) approach, focusing on valence-based encoding differences in either the prefrontal cortex (Dolcos et al., 2004) or the medial temporal lobe (Kensinger \& Schacter, 2006), the extent of valence-based differences in the neural network recruited during encoding is not known. Moreover, it is not known whether valencebased divergence occurs both for the neural processes corresponding with later remembering and with later knowing, or whether the divergence is greater for items that will later be remembered vividly. The goal of the present study, therefore, was to examine the impact of the valence of emotional information on the encoding processes corresponding with later remembering and later knowing.

\section{METHOD}

\section{Participants}

Participants (10 women, 10 men) were all right-handed native English speakers, age $18-30$ years (mean $=23.4$ years), with no history of psychiatric or neurological disorders. All participants had normal or corrected-to-normal vision. Depressed participants and participants taking medication that would affect the central nervous system were excluded from the study. The institutional review board of Boston College approved the behavioral protocol, and the institutional review board from Massachusetts General Hospital approved the neuroimaging procedures.

\section{Materials}

The stimuli were 360 words and 360 pictures. Words were selected from the Affective Norms for English Words (ANEW) database (Bradley \& Lang, 1999). Pictures were taken from the International Affective Picture System database (Lang, Bradley, \& Cuthbert, 1999).

The 360 words were divided by valence (from 1 [negative] to 9 [positive]): 120 negative (mean valence $=2.7), 120$ positive (mean valence $=7.6$ ), and 120 neutral (mean valence $=5.1)$. All neutral words were low in arousal (rated from 1 [calm] to 9 [agitated or excited]; low arousal ratings $<4$ ), and positive and negative words were equally high in arousal (arousal ratings $>5$ ). Positive and negative words were significantly more arousing than neutral words $(p<.001)$, and positive and negative words did not differ from one another in arousal $(p>.25)$. Positive, negative, and neutral words were matched by frequency in the English language, word length, imagibility, and familiarity (as reported in the ANEW database [Bradley \& Lang, 1999] and in the MRC database [Coltheart, 1981]). Positive, negative, and neutral words were selected to be similar in category membership and in interrelatedness (all $\mathrm{ps}>$ .25), as determined by available normative data (Battig \& Montague, 1969; Van Overschelde, Rawson, \& Dunlosky, 2004) and by ratings acquired in a separate group of 10 young adults.

The 360 pictures were also selected to represent three valence categories (also rated from 1 [negative] to 9 [positive]): 120 negative $($ mean valence $=2.7), 120$ positive $($ mean valence $=7.4)$, and 120 neutral (mean valence $=5.2$ ). All neutral pictures were low in arousal (also rated from 1 [calm] to 9 [agitated or excited]; low arousal ratings $<4$ ), and positive and negative pictures were equally high in arousal (arousal ratings $>5$ ). Positive and negative pictures were significantly more arousing than neutral pictures $(p<.001)$, and positive and negative pictures did not differ from one another in arousal $(p>.25)$. Positive, negative, and neutral pictures were matched on visual complexity (normative data taken from a prior investigation; Kensinger \& Schacter, 2006) and brightness (as indicated in Adobe Photoshop [Adobe Systems, San Jose, CA; data from Kensinger \& Schacter, 2006]). Positive, negative, and neutral words were also selected to be similar in category membership (number of images depicting animals, buildings, landscapes, or people). Verbal labels given to picture contexts (e.g., "hospital") also were assessed for relatedness through the use of existing databases (Battig \& Montague, 1969; Van Overschelde et al., 2004) and through ratings provided by a separate group of 10 young adults to ensure that picture interrelatedness was similar for the neural, negative, and positive pictures (all $p s>.20)$.

The stimuli were divided into two lists, each consisting of 180 words and pictures ( 60 from each emotion category). Care was taken to ensure that the items comprising the two lists did not differ in valence or arousal. Items were also matched - as appropriate-for word frequency, word length, imagibility, familiarity, visual complexity, brightness, category membership and interrelatedness, and the number of pictures that included people, animals, buildings, or landscapes. As will be described next, one of these lists served as the study items, whereas the other list served as foils on the recognition test.

\section{Procedure}

Participants were scanned as they viewed one list of 180 words and 180 pictures. This list of stimuli was further broken down into three sets of 60 words and 60 pictures ( 20 from each emotion category) in order to shorten the duration of each individual functional scan. Prior to the presentation of each word or picture, participants saw either the prompt "animate?" or the prompt "common?" which indicated the type of decision the participant was to make about the following stimulus. Participants were instructed that they should respond "yes" in response to the "animate?" prompt if a word or picture depicted something living or described an action that only something animate could execute (e.g., laugh). Participants were instructed that they should respond "yes" in response to a "common?" prompt if a word or picture depicted something that they would encounter in a typical month. The prompt was presented for $500 \mathrm{msec}$; after the prompt, the stimulus was presented for 2,500 $\mathrm{msec}$. After the stimulus disappeared, the participants indicated - via a buttonpress - their "yes" or "no" decision in response to the prompt that had preceded the stimulus. Stimuli were pseudorandomly intermixed with regard to type (words or pictures), emotion (positive, negative, or neutral), and task (animate, common). Interstimulus intervals (ISIs) were jittered, ranging from 4 to $14 \mathrm{sec}$ (Dale, 1999).

About 30 min later, participants underwent a surprise recognition test outside of the scanner. At test, participants were shown a series of words and pictures on a computer screen and were asked to indicate whether each item was one that they had seen in the scanner. If par- 
Table 1

Means and Standard Errors for the Proportion of "Remember" and "Know" Responses Given to Old Items (Hits) and to New Items (False Alarms), and for the Reaction Time (RT, in Milliseconds) to Make Each Response, As a Function of Valence

\begin{tabular}{|c|c|c|c|c|c|c|c|c|c|c|c|c|c|c|c|c|}
\hline \multirow[b]{3}{*}{ Valence } & \multicolumn{8}{|c|}{ Hits } & \multicolumn{8}{|c|}{ False Alarms } \\
\hline & \multicolumn{4}{|c|}{ "Remember" } & \multicolumn{4}{|c|}{ "Know" } & \multicolumn{4}{|c|}{ "Remember" } & \multicolumn{4}{|c|}{ "Know" } \\
\hline & $M$ & $S E$ & RT & $S E$ & $M$ & $S E$ & RT & $S E$ & $M$ & $S E$ & RT & $S E$ & $M$ & $S E$ & RT & $S E$ \\
\hline Negat & .28 & .02 & 1,828 & 55 & .15 & .02 & 213 & 62 & .02 & 0 & 1,982 & 61 & .04 & .01 & 2,142 & 73 \\
\hline Positive & .29 & .02 & 1,876 & 61 & .14 & .02 & 2,007 & 68 & .02 & .01 & 1,926 & 72 & .04 & .01 & 2,121 & 66 \\
\hline Neutral & .26 & .02 & 1,855 & 59 & .10 & .02 & 2,019 & 59 & .01 & 0 & 2,003 & 69 & .03 & .01 & 2,148 & 71 \\
\hline
\end{tabular}

ticipants indicated that an item had been seen, they then were asked whether the item was vividly "remembered" or just "known" to be familiar. They also were asked to specify whether they had been asked to judge the item's animacy or commonness, although responses to this question will not be considered in the present article.

This 30-min delay interval was chosen in order to increase the likelihood of equating the proportion of "remember" and "know" responses given to positive, negative, and neutral items. Pilot data had revealed that at long delays (e.g., of a couple of days), negative pictures were more likely to be "remembered" than positive or neutral pictures, whereas positive pictures were more likely to be "known" (consistent with Ochsner, 2000). However, at the shorter delay, negative, positive, and neutral pictures had more comparable rates of "remembering" and "knowing." Neural differences can be difficult to interpret when there also are behavioral differences, and we wanted to ensure that we had similar power to detect processes associated with "remembering" versus "knowing" for the negative, positive, and neutral items. Therefore, we chose to assess memory after the 30-min delay.

There were 360 words and pictures present at test: a third from each emotion category (positive, negative, and neutral). Of those, 180 words and pictures had been studied, and 180 words and pictures were new. Studied and new items were intermixed randomly. The recognition list was constant for all participants. The particular stimuli that were studied and the condition in which the items were studied (i.e., with either the animate or the common task) were counterbalanced across participants.

\section{Image Acquisition and Preprocessing}

Data were acquired on a 1.5 Tesla Siemens whole-body MRI system (Erlangen, Germany) with a standard birdcage head coil. The stimuli were projected from a Macintosh iBook G4 to a Sharp200 color LCD projector through a collimating lens (Buhl Optical) that projected onto a screen mounted in the magnet bore. Participants viewed the screen through mirrors located on the head coil.

Anatomic images were acquired with a multiplanar rapidly acquired gradient echo (MP-RAGE) sequence (repetition time $[\mathrm{TR}]=$ $2,730 \mathrm{msec}$; echo time $[\mathrm{TE}]=3.31 \mathrm{msec}$; flip angle $=40^{\circ}$; field of view $=256 \times 256 \mathrm{~mm}$; acquisition matrix $=256 \times 256$; slice thickness $=1.33 \mathrm{~mm}$, no gap; $1 \times 1 \times 1.33 \mathrm{~mm}$ resolution). Coplanar and high-resolution T1-weighted localizer images were acquired. In addition, a T1-weighted inversion recovery echo planar image was acquired for auto-alignment.

Functional images were acquired via a $\mathrm{T} 2{ }^{*}$-weighted echo planar imaging sequence that was sensitive to blood oxygenation-dependent contrast (BOLD), with a TR of 3,000 msec, an effective TE of $40 \mathrm{msec}$, and a flip angle of $90^{\circ}$. Twenty-nine interleaved axial-oblique (parallel to the AC-PC line) slices were collected in a $3.125 \times 3.125 \times 3.72 \mathrm{~mm}$ matrix, with a $3.12-\mathrm{mm}$ thickness and a $0.6-\mathrm{mm}$ skip between slices.

Preprocessing and data analysis were completed using SPM2 (statistical parametric mapping; Wellcome Department of Cognitive Neurology, London). Preprocessing included slice time correction, motion correction (using a six-parameter, rigid body transformation algorithm by SPM2), normalization to the Montreal Neurological Institute template (resampling at 3-mm isotropic resolution), and spatial smoothing (at a 7.6-mm isotropic Gaussian kernel).

\section{Event-Related fMRI Data Analysis}

Events were divided by emotion category (positive, negative, or neutral), item type (words or pictures), and subsequent item memory (remembered vividly, known as familiar, or forgotten). Event-related analyses for these event types were completed using the general linear model approach: For each participant, on a voxel by voxel basis, event types were modeled through convolution with a canonical hemodynamic response function. Doing so yielded $\beta$ weights for each voxel. For each statistical contrast, a voxel's activation was considered to differ between two conditions when the difference in $\beta$ weights was statistically positive - as determined by a one-tailed paired $t$ test, with variance estimated using random-effect group analyses. We report the results of contrast analyses including regions that consisted of at least 5 contiguous voxels that were all active at a threshold of $p<.001$. Using the masking function within SPM2, we also examined the regions that were associated with one contrast (at a threshold of $p<.001$ and a 5-voxel extent), but not with one or more other contrasts (even with the threshold reduced to $p<.01$ ). ${ }^{1}$

All activations are presented in neurological coordinates and are displayed on canonical images provided within SPM2. Voxel coordinates are reported in Talairach space (Talairach \& Tournoux, 1988). These coordinates reflect the most significant voxel in each cluster. Event-related time courses were extracted from active clusters by creating ROI, including all significant voxels within an 8-mm radius of each chosen maximum voxel, using the MarsBaR toolbox implemented in SPM (Brett, Anton, Valabregue, \& Poline, 2002). Within each of these ROIs, a hemodynamic response function was calculated for each individual participant and for each condition type (relative to fixation baseline) as a function of peristimulus time $(0-21 \mathrm{sec})$. Statistics were performed on the peak of the signal change within peristimulus time (3-9 sec). These signal change values are displayed in the figures, and the results of ANOVAs and $t$ tests conducted on these signal change values are reported in the figures and tables. In particular, we analyzed the signal change in these regions in order to confirm the main effect of subsequent memory type ( $F$ values reported in Table 2 ) or the interaction between valence and subsequent memory type ( $F$ values reported in Tables 3-5).

\section{RESULTS}

\section{Behavioral Results}

A repeated measures ANOVA was conducted on the corrected recognition scores, with the factors emotion (positive, negative, or neutral) and subsequent memory response ("remember" or "know"); see Table 1. Corrected recognition scores were computed by subtracting the false alarm rate (saying "old" to a new item) from the hit rate (saying "old" to an old item). There was a significant effect of both emotion [signifying better memory for the positive and the negative items as compared with the neutral items; $\left.F(2,40)=28.6, p<.0001, \eta_{\mathrm{p}}^{2}=.588\right]$ and subsequent item memory [with more "remember" than "know" responses given to the items; $\left.F(1,20)=17.91, p<.0001, \eta_{\mathrm{p}}^{2}=.472\right]$. 
Table 2

Regions Implicated in Later Recognition (Recognized > Forgotten), Collapsing Across Valence Types (Positive, Negative, and Neutral)

\begin{tabular}{|c|c|c|c|c|c|c|}
\hline Region & Pattern & $\begin{array}{c}\text { ROI Level } \\
\text { Statistic }\end{array}$ & $\begin{array}{c}\text { ROI } \\
\text { Size } \\
\text { (Voxels) }\end{array}$ & Hemi. & $\begin{array}{c}\text { Talairach } \\
\text { Coordinates } \\
(x, y, z)\end{array}$ & $\begin{array}{c}\text { Approx. } \\
\text { Brodmann } \\
\text { Area }\end{array}$ \\
\hline Medial frontal gyrus & $\mathrm{R}>\mathrm{M}$ & $F(1,19)=18.55$ & 12 & $\mathrm{~L}$ & $-14,6,50$ & 6 \\
\hline \multirow{5}{*}{ Inferior frontal gyrus } & $\mathrm{R}>\mathrm{M}$ & $F(1,19)=19.26$ & 14 & $\mathrm{R}$ & $40,24,-18$ & 47 \\
\hline & $\mathrm{R}>\mathrm{M}$ & $F(1,19)=18.02$ & 15 & $\mathrm{R}$ & $16,-22,-30$ & 45 \\
\hline & $\mathrm{R}>\mathrm{M} \& \mathrm{~K}>\mathrm{M}$ & $F(1,19)=74.01$ & 5 & $\mathrm{R}$ & $54,14,26$ & 9 \\
\hline & $\mathrm{R}>\mathrm{M}$ & $F(1,19)=17.33$ & 25 & $\mathrm{~L}$ & $-30,26,-16$ & 47 \\
\hline & $\mathrm{R}>\mathrm{M} \& \mathrm{~K}>\mathrm{M}$ & $F(1,19)=63.75$ & 6 & $\mathrm{~L}$ & $-46,42,-2$ & 47 \\
\hline \multirow{2}{*}{ Anterior cingulate gyrus } & $\mathrm{R}>\mathrm{M} \& \mathrm{~K}>\mathrm{M}$ & $F(1,19)=22.23$ & 20 & $\mathrm{~L}$ & $-4,2,-4$ & 25 \\
\hline & $\mathrm{R}>\mathrm{M} \& \mathrm{~K}>\mathrm{M}$ & $F(1,19)=98.91$ & 24 & $\mathrm{R}$ & $12,24,18$ & 32 \\
\hline Parahippocampal gyrus & $\mathrm{R}>\mathrm{M} \& \mathrm{~K}>\mathrm{M}$ & $F(1,19)=63.89$ & 31 & $\mathrm{R}$ & $16,-42,2$ & 30 \\
\hline Inferior temporal gyrus & $\mathrm{R}>\mathrm{M} \& \mathrm{~K}>\mathrm{M}$ & $F(1,19)=35.08$ & 43 & $\mathrm{R}$ & $54,-54,-12$ & 20 \\
\hline Superior temporal gyrus & $\mathrm{R}>\mathrm{M} \& \mathrm{~K}>\mathrm{M}$ & $F(1,19)=35.26$ & 34 & $\mathrm{~L}$ & $-38,-52,20$ & 22 \\
\hline \multirow[t]{3}{*}{ Fusiform gyrus } & $\mathrm{R}>\mathrm{M} \& \mathrm{~K}>\mathrm{M}$ & $F(1,19)=108.70$ & 7 & $\mathrm{~L}$ & $-48,-34,-18$ & 20 \\
\hline & $\mathrm{R}>\mathrm{M}$ & $F(1,19)=21.17$ & 5 & $\mathrm{R}$ & $42,-64,-10$ & 37 \\
\hline & $\mathrm{R}>\mathrm{M} \& \mathrm{~K}>\mathrm{M}$ & $F(1,19)=78.94$ & 9 & $\mathrm{R}$ & $48,-42,-14$ & 37 \\
\hline Inferior parietal lobule & $\mathrm{K}>\mathrm{M}$ & $F(1,19)=17.77$ & 22 & $\mathrm{R}$ & $30,-42,52$ & 40 \\
\hline \multirow[t]{2}{*}{ Cuneus/precuneus } & $\mathrm{K}>\mathrm{M}$ & $F(1,19)=30.58$ & 6 & $\mathrm{R}$ & $20,-76,34$ & 7 \\
\hline & $\mathrm{R}>\mathrm{M}$ & $F(1,19)=28.96$ & 7 & $\mathrm{R}$ & $18,-48,40$ & 7 \\
\hline Hippocampus & $\mathrm{R}>\mathrm{M}$ & $F(1,19)=66.90$ & 9 & $\mathrm{R}$ & $20,-12,-16$ & \\
\hline Caudate & $\mathrm{R}>\mathrm{M}$ & $F(1,19)=57.37$ & 7 & $\mathrm{R}$ & $24,-26,24$ & \\
\hline \multirow[t]{3}{*}{ Cerebellum } & $\mathrm{R}>\mathrm{M} \& \mathrm{~K}>\mathrm{M}$ & $F(1,19)=46.51$ & 8 & $\mathrm{R}$ & $52,-56,-28$ & \\
\hline & $\mathrm{R}>\mathrm{M} \& \mathrm{~K}>\mathrm{M}$ & $F(1,19)=20.80$ & 23 & $\mathrm{R}$ & $46,-74,-20$ & \\
\hline & $\mathrm{R}>\mathrm{M} \& \mathrm{~K}>\mathrm{M}$ & $F(1,19)=39.89$ & 10 & $\mathrm{~L}$ & $-44,-68,-24$ & \\
\hline
\end{tabular}

Note-Hemi., hemisphere. $F$ values refer to the main effect of later recognition (recognized vs. forgotten). All $F$ values are significant at $p<.001$. For each coordinate, the pattern is indicated (remembered $[\mathrm{R}]>$ missed $[\mathrm{M}]$ or known $[\mathrm{K}]>$ missed $[\mathrm{M}]$ ); these patterns were determined by an ANOVA conducted with three levels of recognition response (remembered, known, or missed) and by subsequent $t$ tests.

There was no interaction between the two factors, and a main effect of emotion existed both when "remember" responses were analyzed and when "know" responses were analyzed separately (both $p$ s $<.05$ ).

A repeated measures ANOVA was also conducted on the reaction times (RTs) to make the "old" decision at retrieval, with the factors emotion (positive, negative, or neutral), item type (old or new), and subsequent memory response ("remember" or "know"); see Table 1. This ANOVA revealed a main effect of item type ["old" responses were given faster to old items (i.e., hits) than to new items (i.e., false alarms); $F(1,20)=8.31, p<.01]$ and a main effect of item memory [with participants being faster to give "old" responses to items they "remembered" than to items they "knew"; $F(1,20)=6.51, p<.05]$. Importantly, there also was an interaction between emotion, item type, and item memory $[F(2,40)=7.31, p<$ $.05]$. The discrepancy in RT between "remembered" and "known" items was greater for negative hits $(307 \mathrm{msec})$ than for any other response (e.g., for negative false alarms [160 msec] or for positive hits [131 msec]).

A repeated measures ANOVA conducted on participants' RTs during the encoding task - with emotion (positive, negative, or neutral) and task (animate or common)

Table 3

Regions Implicated in Later Recognition (Recognized > Forgotten), for Positive and Negative Items but Not for Neutral Items

\begin{tabular}{|c|c|c|c|c|c|c|}
\hline Region & Pattern & $\begin{array}{c}\text { ROI Level } \\
\text { Statistic }\end{array}$ & $\begin{array}{l}\text { ROI } \\
\text { Size }\end{array}$ & Hemi. & $\begin{array}{c}\text { Talairach } \\
\text { Coordinates } \\
(x, y, z)\end{array}$ & $\begin{array}{c}\text { Approx. } \\
\text { Brodmann } \\
\text { Area }\end{array}$ \\
\hline Inferior/orbital frontal gyrus & $\mathrm{R}>\mathrm{M} \& \mathrm{~K}>\mathrm{M}$ & $F(2,18)=18.09$ & 24 & $\mathrm{~L}$ & $-28,38,-4$ & $10 / 11 / 47$ \\
\hline \multirow[t]{2}{*}{ Parahippocampal gyrus } & $\mathrm{R}>\mathrm{M} \& \mathrm{~K}>\mathrm{M}$ & $F(2,18)=14.68$ & 13 & $\mathrm{~L}$ & $-38,-34,-10$ & 36 \\
\hline & $\mathrm{R}>\mathrm{M}$ & $F(2,18)=24.78$ & 5 & $\mathrm{R}$ & $42,-36,-8$ & 36 \\
\hline Amygdala & $\mathrm{R}>\mathrm{M} \& \mathrm{~K}>\mathrm{M}$ & $F(2,18)=21.40$ & 11 & $\mathrm{~L}$ & $-12,-2,-14$ & \\
\hline Caudate & $\mathrm{R}>\mathrm{M}$ & $F(2,18)=32.57$ & 5 & $\mathrm{R}$ & $22,-8,28$ & \\
\hline \multirow{2}{*}{ Thalamus } & $\mathrm{R}>\mathrm{M} \& \mathrm{~K}>\mathrm{M}$ & $F(2,18)=46.58$ & 5 & $\mathrm{R}$ & $22,-24,0$ & \\
\hline & $\mathrm{R}>\mathrm{M} \& \mathrm{~K}>\mathrm{M}$ & $F(2,18)=9.28$ & 24 & $\mathrm{~L}$ & $-8,-24,18$ & \\
\hline \multirow[t]{2}{*}{ Cerebellum } & $\mathrm{R}>\mathrm{M} \& \mathrm{~K}>\mathrm{M}$ & $F(2,18)=17.25$ & 76 & $\mathrm{R}$ & $8,-36,-22$ & \\
\hline & $\mathrm{R}>\mathrm{M} \& \mathrm{~K}>\mathrm{M}$ & $F(2,18)=16.68$ & 15 & $\mathrm{R}$ & $36,-62,-10$ & \\
\hline
\end{tabular}

Note-Hemi., hemisphere. $F$ values refer to the interaction between valence (positive, negative, neutral) and later recognition response (recognized, forgotten). All $F$ values are significant at $p<.001$. For each coordinate, the pattern is indicated (remembered $[\mathrm{R}]>$ missed $[\mathrm{M}]$ or known $[\mathrm{K}]>$ missed $[\mathrm{M}]$ ); these patterns were determined by an ANOVA conducted with three levels of recognition response (remembered, known, or missed) and by subsequent $t$ tests. 
Table 4

Valence Effects on Regions Active for Remembered (R) $>$ Known (K)

\begin{tabular}{|c|c|c|c|c|c|}
\hline Region & $\begin{array}{l}\text { ROI Level } \\
\text { Statistic }\end{array}$ & $\begin{array}{l}\text { ROI } \\
\text { Size }\end{array}$ & Hemi. & $\begin{array}{c}\text { Talairach } \\
\text { Coordinates } \\
(x, y, z)\end{array}$ & $\begin{array}{c}\text { Approx. } \\
\text { Brodmann } \\
\text { Area }\end{array}$ \\
\hline
\end{tabular}

Negative Remembered $>$ Known

Not Positive Remembered $>$ Known or Neutral Remembered $>$ Known

$\begin{array}{llrlcc}\text { Parahippocampal/fusiform gyrus } & F(1,19)=9.50 & 15 & \mathrm{~L} & -28,-28,-20 & 35 / 36 \\ \text { Middle temporal gyrus } & F(1,19)=13.40 & 5 & \mathrm{R} & 46,-58,0 & 37 \\ \text { Inferior temporal gyrus } & F(1,19)=17.75 & 7 & \mathrm{~L} & -46,-58,-4 & 19 \\ & F(1,19)=7.95^{*} & 15 & \mathrm{~L} & -42,-66,0 & 37 \\ \text { Inferior temporal/occipital gyrus } & F(1,19)=14.5 & 26 & \mathrm{~L} & -53,-60,-4 & 19 / 37\end{array}$

Negative Remembered $>$ Known

and Positive Remembered $>$ Known

$\begin{array}{llllll}\text { Inferior/orbital frontal gyrus } & \mathrm{N} / \mathrm{A} & 6 & \mathrm{R} & 32,42,-2 & 10 / 11 / 47\end{array}$

Note-Hemi., hemisphere. $F$ values refer to the interaction between valence (positive, negative) and later recognition response $(\mathrm{R}, \mathrm{K}) . F$ values are significant at $p<.001$ unless otherwise indicated. N/A signifies that the interaction value is not relevant, because regions were selected because of overlapping (rather than distinct) patterns of activation. ${ }^{*} p \leq .01$.

as factors - revealed no main effects nor any interactions. Thus, emotion did not influence participants' RTs on the encoding task.

\section{fMRI Results}

We first examined the regions that were related to the general subsequent-memory effect (i.e., remembered or known $>$ forgotten). Across all valence types, this contrast revealed the standard subsequent-memory network (reviewed by Paller \& Wagner, 2002), including regions of the inferior prefrontal cortex, the hippocampus, and the parahippocampal gyrus (see Table 2). When looking at regions that corresponded with subsequent memory for both the positive and the negative items (but not for the neutral items), we found that regions within the amygdala, orbitofrontal/ventrolateral prefrontal cortex, and parahippocampal gyrus were revealed (see Table 3), consistent with prior studies of emotional memory (reviewed by LaBar \& Cabeza, 2006). Out of all of the regions identified in the analyses, the amygdala was the only region to show any

Table 5

Valence Effects on Regions Active for Known (K) $>$ Remembered (R)

\begin{tabular}{cccccc}
\multicolumn{8}{c}{ Valence Effects on Regions Active for Known $(\mathbf{K})>$ Remembered $(\mathbf{R})$} \\
\hline \\
\multirow{2}{*}{ Region } & ROI Level & ROI & & Talairach & Approx. \\
Statistic & Size & Hemi. & $(x, y, z)$ & Area \\
\hline
\end{tabular}

Positive Known $>$ Remembered

Not Negative Known $>$ Remembered or Neutral Known $>$ Remembered

$\begin{array}{llrlrr}\text { Middle frontal gyrus } & F(1,19)=12.03^{* *} & 11 & \mathrm{R} & 30,10,49 & 6 \\ \text { Inferior frontal gyrus } & F(1,19)=14.70 & 35 & \mathrm{~L} & -16,27,-8 & 47 \\ \text { Anterior cingulate gyrus } & F(1,19)=19.90 & 60 & \mathrm{R} & 4,-7,46 & 24 \\ \text { Posterior cingulate gyrus } & F(1,19)=14.30 & 14 & \mathrm{R} & 10,-34,27 & 23 \\ \text { Postcentral gyrus } & F(1,19)=8.50^{*} & 12 & \mathrm{~L} & -53,-9,23 & 43 \\ \text { Inferior/superior parietal lobule } & F(1,19)=20.10 & 22 & \mathrm{~L} & -34,-48,45 & 7 / 40 \\ \text { Inferior parietal lobule } & F(1,19)=15.58 & 5 & \mathrm{~L} & -46,-37,46 & 40 \\ \text { Superior temporal gyrus } & F(1,19)=8.67^{*} & 5 & \mathrm{~L} & -59,0,-2 & 22 \\ \text { Lentiform nucleus } & F(1,19)=7.76^{*} & 17 & \mathrm{R} & 26,4,11 & \\ \text { Caudate } & F(1,19)=8.77^{*} & 15 & \mathrm{~L} & -12,21,-4 & \end{array}$

Negative Known $>$ Remembered

Not Positive Known $>$ Remembered or Neutral Known $>$ Remembered

\begin{tabular}{lccccc} 
Superior frontal gyrus & $F(1,18)=13.10$ & 6 & $\mathrm{R}$ & $24,8,51$ & 6 \\
Cingulate gyrus & $F(1,19)=7.25^{*}$ & 5 & $\mathrm{~L}$ & $0,-18,27$ & 23 \\
Substantia nigra & $F(1,19)=21.30$ & 9 & $\mathrm{R}$ & $8,-18,-9$ & \\
& \multicolumn{2}{c}{ Negative Known $>$ Remembered } \\
& and Positive Known $>$ Remembered & & \\
Superior frontal gyrus & $\mathrm{N} / \mathrm{A}$ & 7 & Bilateral & $-6,42,55$ & 8 \\
& $\mathrm{~N} / \mathrm{A}$ & 6 & $\mathrm{R}$ & $30,8,51$ & 6 \\
Cingulate gyrus & $\mathrm{N} / \mathrm{A}$ & 5 & $\mathrm{R}$ & $24,-20,32$ & $23 / 31$ \\
Inferior parietal lobule & $\mathrm{N} / \mathrm{A}$ & 7 & $\mathrm{~L}$ & $-38,-24,24$ & 40 \\
\hline
\end{tabular}

Note-Hemi., hemisphere. $F$ values refer to the interaction between valence (negative, positive) and later recognition response $(\mathrm{R}, \mathrm{K}) . F$ values are significant at $p<.001$ unless otherwise indicated. N/A signifies that the interaction value is not relevant, because regions were selected because of overlapping (rather than distinct) patterns of activation. ${ }^{*} p \leq .01 .{ }^{* *} p<.005$. 
evidence of a sex effect. As can be seen in Figure 1, the correspondence to subsequent memory in the left amygdala was marginally stronger for women than it was for men. However, the amygdala's correspondence to subsequent memory was significant in both sexes, and the interaction between subsequent memory (i.e., later recognized vs. forgotten) and sex did not reach significance $(p>.10)$.

Having replicated the results of prior studies examining the effects of emotion on subsequent memory performance, we then went on to examine the impact of emotional valence on the neural processes associated with subsequent "remembering" versus "knowing." These analyses were restricted to items that were associated with successful subsequent recognition, examining how valence influenced the encoding processes that led to later "remembering" versus later "knowing."

In order to examine the regions that were related specifically to the encoding of negative items that were later remembered, we used the masking function within SPM to determine areas that were active in the contrast analysis for negative remembered items $>$ negative known items (at $p<$ $.001)$, but not in the contrasts of neutral remembered items $>$ neutral known items or positive remembered items $>$ positive known items (even at $p<.01$ ). This analysis revealed a network of occipital and temporal regions (see red regions

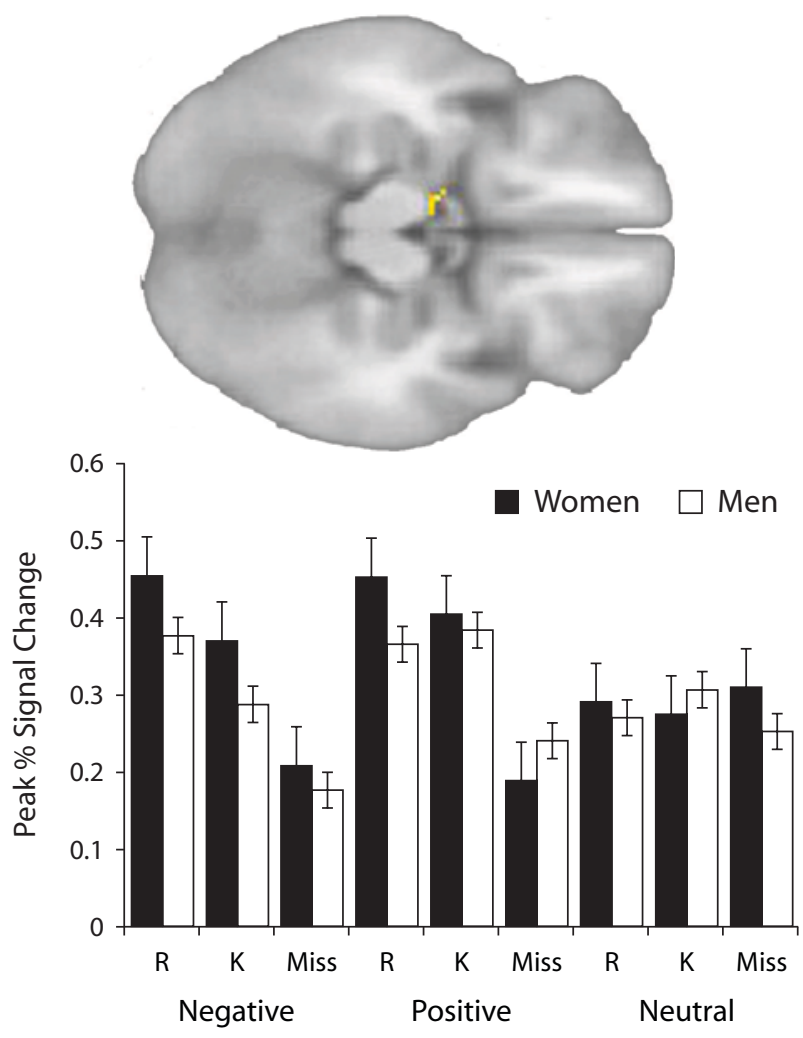

Figure 1. Left amygdala activity was associated with general subsequent memory (remembered or known $>$ forgotten) for the negative and positive items, but not for the neutral items. Its correspondence to subsequent memory was significant in both men and women, although its correspondence was marginally stronger in women than in men (see graph). of Figure 2 and the upper portion of Table 4) previously indicated in visual processing (Nakamura et al., 2000). ${ }^{2}$

There were no regions whose activity related uniquely to the encoding of positive items that would later be remembered; a masking analysis examining areas that were active for the contrast of positive remembered items $>$ positive known items (at $p<.001$ )- but not in the contrasts of neutral remembered items $>$ neutral known items or negative remembered items $>$ negative known items (at $p<.01)$-revealed no active areas.

A conjunction analysis also was conducted to examine the regions that corresponded with later remembering of both negative and positive items (i.e., negative remembered $>$ negative known and positive remembered $>$ positive known). When each contrast was analyzed at $p<.01$ and a 5-voxel extent (i.e., a conjoint probability of $p<.001$ using Fisher's estimate [Fisher, 1950]), no regions were revealed. However, when the threshold of each contrast was lowered to $p<.05$ and a 5 -voxel extent, activity within one region of the inferior prefrontal gyrus (Talairach coordinates: 32, 42, -2 [BA 10/11/47]) was shown to correspond with subsequent "remembering" of both negative and positive items (see the green region in Figure 2).

We then analyzed the regions that were more active during the encoding of items that were later "known" rather than "remembered." Multiple areas were active in the contrast for positive known items $>$ positive remembered items (at $p<.001$ ), but not in the contrasts of neutral known items $>$ neural remembered items or negative known items $>$ negative remembered items (at $p<.01)$. These areas included multiple regions along the cingulate gyrus as well as regions of the frontal and parietal lobes (see the green regions of Figure 3 and the upper portion of Table 5).

Another analysis was conducted to determine the activity involved in the encoding of negative items that were later known (examining areas that were active for the contrast of negative known items $>$ negative remembered items, but not neutral known items $>$ neutral remembered items or positive known items $>$ positive remembered items). This analysis revealed activations along the cingulate gyrus and in the superior frontal lobe (see the red regions of Figure 3 and the middle portion of Table 5). However, these activations were nowhere near as extensive as the activations elicited specifically by the positive pictures that were later known.

A conjunction analysis also was conducted to examine whether there were regions that corresponded with subsequent "knowing" for both the negative and the positive items. When each contrast was analyzed at $p<.01$ and a 5-voxel extent (i.e., a conjoint probability of $p<.001$ using Fisher's estimate [Fisher, 1950]), regions in the cingulate gyrus, superior frontal gyrus, and inferior parietal lobe were identified (see the lower portion of Table 5 and the blue regions of Figure 3).

\section{DISCUSSION}

The results of the present study indicate that there are some valence-independent processes that support "remem- 

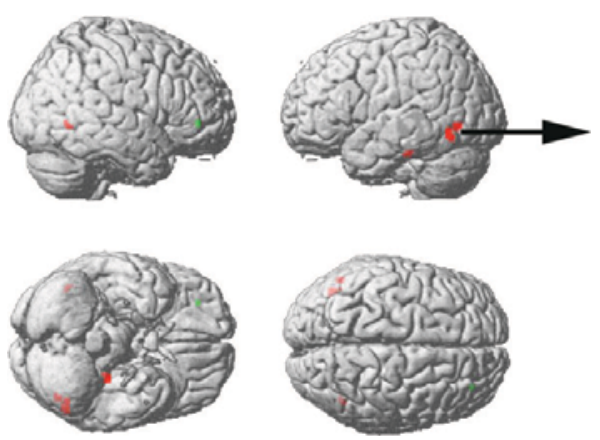

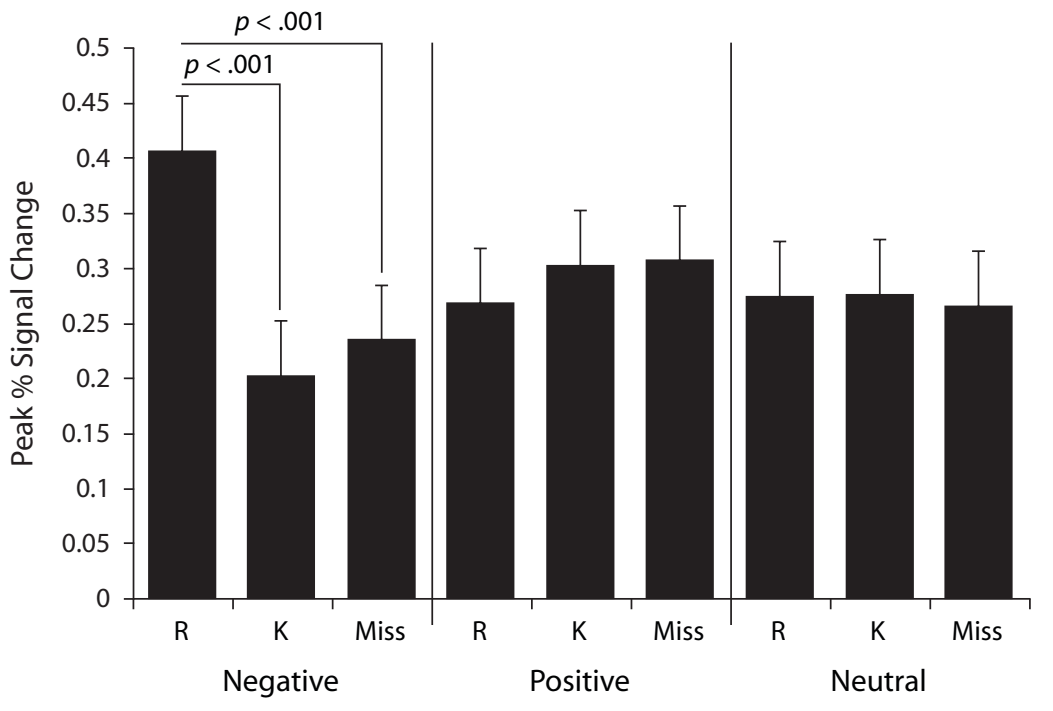

Figure 2. The red regions were disproportionately active for negative items later "remembered" (negative remembered items $>$ negative known items but not positive remembered items $>$ positive known items or neutral remembered items $>$ neutral known items). No regions were disproportionately active for positive items later "remembered," and no regions responded both to negative remembered $>$ known and to positive remembered $>$ known. One region of the right inferior prefrontal cortex (depicted in green) was active for both positive remembered $>$ known and negative remembered $>$ known.

bering" versus "knowing." For both positive and negative items, activity within a region spanning the orbitofrontal and ventrolateral prefrontal cortex corresponded with later "remembering." For both valences of information, activity within the midcingulate gyrus, the inferior parietal lobe, and the superior frontal lobe was associated with "knowing." However, despite these commonalities, valence influenced many of the regions that supported later "remembering" or "knowing." Activity within temporo-occipital regions was associated specifically with "remembering" negative items, and activity within expansive regions of the prefrontal cortex, the cingulate gyrus, and the parietal lobe corresponded specifically with "knowing" positive items. Next, we discuss the implications of these results.

The fact that the orbitofrontal cortex corresponded with "remembering" fits well within the broader emotional memory literature. A number of studies have revealed that activity within the orbitofrontal and ventrolateral prefrontal cortex corresponds with later memory for episodic detail (reviewed by Petrides, 2007) and that this region's correspondence to subsequent memory can be particularly strong for items with emotional content (see, e.g.,

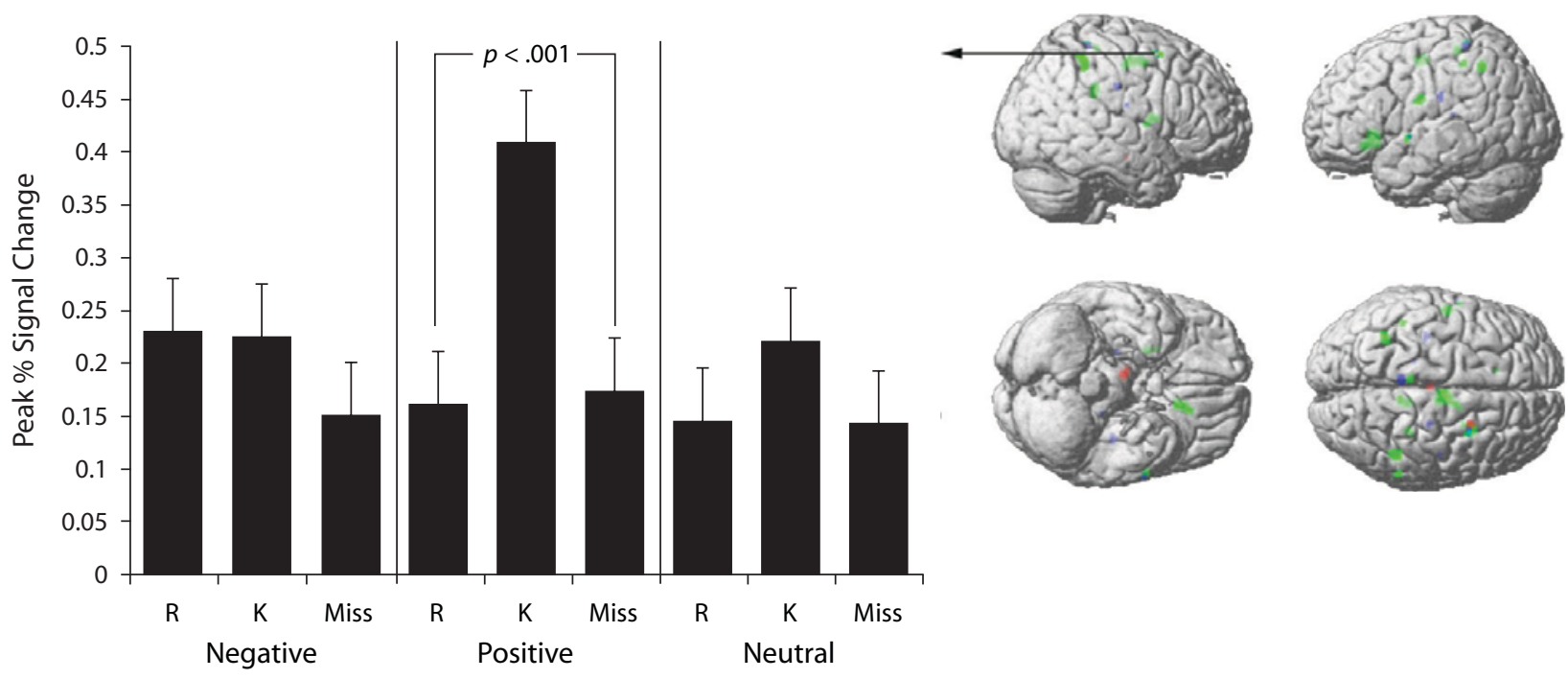

Figure 3. The red regions were disproportionately active for negative items later "known" (negative known > negative remembered items, but not positive known items $>$ positive remembered items or neutral known items $>$ neutral remembered items). The green regions were disproportionately active for positive items that were later "known." The blue regions corresponded both to positive known $>$ positive remembered and also to negative known $>$ negative remembered. 
Kensinger \& Schacter, 2005). Thus, it makes sense that activity within the orbitofrontal cortex would correspond with later "remembering" of episodic detail. These prior studies, however, had not examined the effects of valence on this region's tie to subsequent memory. Instead, they either had focused exclusively on negative information or had combined data from positive and negative items. Thus, the present study is the first to suggest that the orbitofrontal cortex supports later "remembering" for both negative and positive items.

The regions that supported later "knowing" of positive and negative items - regions of the midcingulate gyrus, superior frontal gyrus, and inferior parietal lobealso make sense when considered in the context of other memory studies. These regions are often implicated in the retrieval of semantic and episodic information, including self-referential information (see, e.g., Johnson et al., 2002; Northoff \& Bermpohl, 2004; Northoff et al., 2006). Although at first it may seem counterintuitive that these regions would be associated with "knowing" rather than with "remembering," in fact, these regions previously have been found to correspond with the encoding of items for which people forget either the specific details (Garoff, Slotnick, \& Schacter, 2005) or the items altogether (Otten \& Rugg, 2001; Wagner \& Davachi, 2001). For example, Garoff et al. (2005) compared the neural correlates of specific, detailed memory (saying "same" to an item that is exactly the same) to that of more general memory (forgetting the details: saying "same" to an item that in fact is only similar to a studied item or "similar" to an item that in fact is the same as the studied item). A number of the regions that Garoff et al. found to correspond with general rather than with specific recognition overlap with the regions that were revealed in the present study to be associated with later "knowing." In particular, both that prior study and the present one revealed activity in inferior parietal and cingulate regions. Similarly, Wagner and Davachi, and Otten and Rugg, found that subsequent forgetting was associated with activity in the posterior cingulate gyrus and in the medial and inferior parietal lobe. Thus, there are common regions of the inferior and medial parietal lobe and the cingulate gyrus that are implicated both in the retrieval of semantic and episodic detail (Buckner, Koutstaal, Schacter, Wagner, \& Rosen, 1998; Demb et al., 1995; Slotnick, Moo, Segal, \& Hart, 2003; Wagner, ParéBlagoev, Clark, \& Poldrack, 2001; Wheeler \& Buckner, 2003) and in the poor encoding of contextual details.

This overlap between areas related to the retrieval of semantic and episodic information and the forgetting of details might reflect the fact that these retrieval-related processes are engaged at the cost of other encoding-related processes. If you see a picture of a smiling baby and you begin to think about your daughter's first birthday, it is entirely possible that you will cease to pay attention to the picture and thus will be left with a less detailed memory of its presentation. Thus, the increase in elaborative processing (e.g., retrieval of semantic or autobiographical associations) for any type of information may sometimes come at the expense of encoding episodic details associated with the item's presentation.
Although an engagement of these sorts of processes occurred for both positive and negative items, the encoding of positive items later "known" was associated with disproportionate activity within regions along the cingulate gyrus, within the parietal lobe, and in the prefrontal cortex. This valence-specific recruitment may suggest that, although all items may occasionally evoke encoding processes that distract from the ability to encode episodic details that will later allow a person to "remember" the photos, this type of processing may occur more often for the positive items.

This hypothesis is consistent with studies that have found that positive emotion leads to a broadening of attention and cognitive flexibility (Fredrickson \& Branigan, 2005; Rowe, Hirsh, \& Anderson, 2007). People in positive moods tend to process information in a holistic and associative nature, but often miss the local details (see Fredrickson, 2004; Gasper \& Clore, 2002). These behavioral findings are consistent with the results of the present study, suggesting that the processing of positive information is associated with the increased engagement of processes that allow a person to later "know" that an item is familiar, but not to "remember" the episodic details of its presentation.

In contrast with the network of regions specifically associated with "knowing" positive items, no region corresponded specifically with "remembering" positive items. There were, however, a number of regions that were associated specifically with the "remembering" of negative items. All of the regions identified were within temporo-occipital sensory processing regions. These findings suggest that there may be something different about the recollective experience associated with negative items as compared with positive or neutral ones: Negative items may be "remembered" with more sensory detail than would positive or neutral items. This hypothesis seems particularly plausible in light of studies revealing that negative arousing items often are remembered with more visual details than are either positive arousing or neutral items (Kensinger, Garoff-Eaton, \& Schacter, 2006, 2007a, 2007b), and that memory for visual details may recruit fusiform processes (Kensinger, Garoff-Eaton, \& Schacter, 2007c). More broadly, these results are consistent with behavioral data suggesting that negative emotion may be associated with a detail-oriented processing mode, leading individuals to focus on both item-specific features and local stimulus details (reviewed by Anderson, 2005; Gasper, 2004; Gasper \& Clore, 2002; Reisberg \& Hertel, 2004).

Importantly, note that in the present study, these valence-specific neural differences were revealed in the context of a recognition memory task in which the overall recognition rates - and the proportion of "remember" and "know" responses - were equated between the positive and negative items. Therefore, simple differences in either discrimination or the power to detect subsequently "remembered" versus "known" items cannot explain the results. Rather, the differences seem to reflect true divergence in the processes engaged during the successful encoding of negative and positive items. Even though the behavioral memory data do not reveal differences based 
on valence, the neural data make clear that such differences are present. In other words, although negative and positive items can be "remembered" or "known" equally often, the processes that lead to their "remembering" and "knowing" are different.

Of course, it would be surprising if such neural differences never conveyed behavioral effects. Indeed, there is behavioral evidence to suggest that the "remembering" and "knowing" of positive and negative items may not be identical. The most convincing evidence for such a divergence has come from studies examining the durability of "remember" versus "know" responses for positive and negative items. These studies often reveal that negative items tend to be "remembered" even after long delays, whereas the ability for participants to "remember" positive items tends to decrease after longer delays. Thus, when memory is assessed after lengthy delays (e.g., of a few days or more), rates of "remembering" tend to be higher for negative than for positive items, whereas positive items are more likely to be "known" (see, e.g., LaBar \& Phelps, 1998; Ochsner, 2000). This pattern also was revealed with the stimuli used in the present study: Our pilot testing revealed that when participants' memory was tested a couple of days later, rates of "remembering" were reliably higher for the negative items than for the positive items (unpublished raw data, Kensinger, 2007). Even with the shorter delay used here, there was some evidence of behavioral divergence in the RTs to perform the recognition memory task. Although participants were always faster to indicate that "remembered" items were old than to indicate that "known" items were old, this discrepancy in RT was greatest for the negative items. This observation fits well with the neuroimaging data, suggesting that participants' "remember" responses for negative items may have been made on the basis of more information - or on different types of information - than their "remember" responses for positive or neutral items.

In summary, the results of the present study suggest that the valence of emotional information has an impact on the encoding processes related to later "remembering" versus "knowing." Although "remembering" of both negative and positive items is associated with activity within the orbitofrontal cortex, subsequent "remembering" of negative items is associated with additional sensory processing not associated with the subsequent "remembering" of positive information. This finding suggests that much of what people "remember" about negative items may be the sensory details that were encoded. By contrast, later "knowing" was associated with activity in the midcingulate gyrus, superior frontal cortex, and inferior parietal lobe. Although both negative and positive items recruited these regions during encoding, there was a more expansive recruitment of this network that predicted "knowing" of positive items as compared with negative ones. We suggest that by engaging these processes that are associated with semantic and episodic retrieval and with self-referential processing, participants may increase the likelihood that they extract at least some general information about positive stimuli, but they may simultaneously decrease the likelihood that they process the episodic details that would be required to support later "remembering."

\section{AUTHOR NOTE}

This research was supported by Grant BCS-0542694 to E.A.K. from the National Science Foundation and by a National Defense Science and Engineering graduate fellowship to K.R.M. We thank Keely Muscatell for comments on an earlier version of the manuscript. Correspondence should be addressed to K. R. Mickley, Boston College, McGuinn Hall, 140 Commonwealth Ave., Chestnut Hill, MA 02467 (e-mail: mickley@bc.edu).

\section{REFERENCES}

Adolphs, R., Tranel, D., \& Buchanan, T. W. (2005). Amygdala damage impairs emotional memory for gist but not details of complex stimuli. Nature Neuroscience, $\mathbf{8}, 512-518$.

Anderson, A. K. (2005). Affective influences on the attentional dynamics supporting awareness. Journal of Experimental Psychology: General, 134, 258-261.

Battig, W. F., \& Montague, W. E. (1969). Category norms for verbal items in 56 categories: A replication and extension of the Connecticut category norms. Journal of Experimental Psychology, 80, 1-46.

Bless, H., Schwarz, N., \& Wieland, R. (1996). Mood and the impact of category membership and individuating information. European Journal of Social Psychology, 26, 935-959.

Bradley, M. M., \& LANG, P. J. (1999). Affective norms for English words (ANEW): Stimuli, instruction manual and affective ratings (Tech. Rep. No. C-1). Gainesville: University of Florida, Center for Research in Psychophysiology.

Brett, M., Anton, J.-L., Valabregue, R., \& Poline, J.-B. (2002, June). Region of interest analysis using an SPM toolbox. Poster presented at the 8th International Conference on Functional Mapping of the Human Brain, Sendai, Japan. Abstract available on CD-ROM in NeuroImage, 16.

Buckner, R. L., Koutstaal, W., Schacter, D. L., Wagner, A. D., \& Rosen, B. R. (1998). Functional-anatomic study of episodic retrieval using fMRI. NeuroImage, 7, 151-162.

Coltheart, M. (1981). The MRC psycholinguistic database. Quarterly Journal of Experimental Psychology, 33A, 497-505.

DALE, A. M. (1999). Optimal experimental design for event-related fMRI. Human Brain Mapping, 8, 109-114.

Davachi, L., \& Wagner, A. D. (2002). Hippocampal contributions to episodic encoding: Insights from relational and item-based learning. Journal of Neurophysiology, 88, 982-990.

DAvidson, P. S. R., \& GLiSKY, E. L. (2002). Is flashbulb memory a special instance of source memory? Evidence from older adults. Memory, 10, 99-111.

Demb, J. B., Desmond, J. E., Wagner, A. D., Vaidya, C. J., Glover, G. H., \& Gabrieli, J. D. (1995). Semantic encoding and retrieval in the left inferior prefrontal cortex: A functional MRI study of task difficulty and process specificity. Journal of Neuroscience, 15, 5870-5878.

Dobbins, I. G., Kroll, N. E. A., \& Yonelinas, A. P. (2004). Dissociating familiarity from recollection using note rehearsal. Memory \& Cognition, 32, 932-944.

Dolcos, F., LaBar, K. S., Cabeza, R. (2004). Dissociable effects of arousal and valence on prefrontal activity indexing emotional evaluation and subsequent memory: An event-related fMRI study. NeuroImage, 23, 64-74.

FISHER, R. A. (1950). Statistical methods for research workers. London: Oliver and Boyd.

Fredrickson, B. L. (2004). Neural correlates of self-reflection. Philosophical Transactions of the Royal Society of London: Series B, 125, 1808-1814.

Fredrickson, B. L., \& Branigan, C. (2005). Positive emotions broaden the scope of attention and thought-action repertoires. Cognition \& Emotion, 19, 313-332.

Garoff, R. J., Slotnick, S. D., \& Schacter, D. L. (2005). The neural origins of specific and general memory: The role of the fusiform cortex. Neuropsychologia, 43, 847-859.

GASPER, K. (2004). Do you see what I see? Affect and visual information processing. Cognition \& Emotion, 18, 405-421.

Gasper, K., \& Clore, G. L. (2002). Attending to the big picture: Mood and global versus local processing of visual information. Psychological Science, 13, 34-40. 
JACOBY, L. L. (1991). A process dissociation framework: Separating automatic from intentional uses of memory. Journal of Memory \& Language, 30, 513-541.

Johnson, S. C., Baxter, L. C., Wilder, L. S., Pipe, J. G., Heiserman, J. E., \& Prigatano, G. P. (2002). Neural correlates of self-reflection. Brain, 125, 1808-1814.

Kensinger, E. A., Clarke, R. J., \& Corkin, S. (2003). What neural correlates underlie successful encoding and retrieval? A functional magnetic resonance imaging study using a divided attention paradigm. Journal of Neuroscience, 23, 2407-2415.

Kensinger, E. A., \& Corkin, S. (2004). Two routes to emotional memory: Distinct neural processes for valence and arousal. Proceedings of the National Academy of Sciences, 101, 3310-3315.

Kensinger, E. A., Garoff-Eaton, R. J., \& Schacter, D. L. (2006). Memory for specific visual details can be enhanced by negative arousing content. Journal of Memory \& Language, 54, 99-112.

Kensinger, E. A., Garoff-Eaton, R. J., \& Schacter, D. L. (2007a). Effects of emotion on memory specificity in young and older adults. Journals of Gerontology, 62B, 208-215.

Kensinger, E. A., Garoff-Eaton, R. J., \& Schacter, D. L. (2007b). Effects of emotion on memory specificity: Memory trade-offs elicited by negative visually arousing stimuli. Journal of Memory \& Language, 56, 575-591.

Kensinger, E. A., Garoff-Eaton, R. J., \& Schacter, D. L. (2007c). How negative emotion enhances the visual specificity of a memory. Journal of Cognitive Neuroscience, 19, 1872-1887.

Kensinger, E. A., \& Schacter, D. L. (2005). Emotional content and reality-monitoring ability: fMRI evidence for the influence of encoding processes. Neuropsychologia, 43, 1429-1443.

Kensinger, E. A., \& Schacter, D. L. (2006). Amygdala activity is associated with the successful encoding of item, but not source, information for positive and negative stimuli. Journal of Neuroscience, 26, 2564-2570

Knowlton, B. J., \& Squire, L. R. (1995). Remembering and knowing: Two different expressions of declarative memory. Journal of Experimental Psychology: Learning, Memory, \& Cognition, 21, 699-710.

LABAR, K. S., \& CABEZA, R. (2006). Cognitive neuroscience of emotional memory. Nature Reviews Neuroscience, 7, 54-64.

LaBAR, K. S., \& Phelps, E. A. (1998). Arousal-mediated memory consolidation: Role of the medial temporal lobe in humans. Psychological Science, 9, 490-493.

Lang, P. J., Bradley, M. M., \& Cuthbert, B. N. (1999). International affective picture system (IAPS): Technical manual and affective ratings. Gainesville: University of Florida, Center for Research in Psychophysiology.

MANDLER, G. (1980). Recognizing: The judgment of previous occurrence. Psychological Review, 87, 252-271.

Manns, J. R., Hopkins, R. O., Reed, J. M., Kitchener, E. G., \& SQUIRE, L. R. (2003). Recognition memory and the human hippocampus. Neuron, 37, 171-180.

Nakamura, K., Kawashima, R., Sato, N., NaKamura, A., SugiURA, M., KATO, T., ET AL. (2000). Functional delineation of the human occipito-temporal areas related to face and scene processing. Brain, 123, 1903-1912.

NorthofF, G., \& Bermpohl, F. (2004). Cortical midline structures and the self. Trends in Cognitive Sciences, 8, 102-107.

Northoff, G., Heinzel, A., De Greck, M., Bermpohl, F., DobrowOLNY, H., \& PANKSEPP, J. (2006). Self-referential processing in our brain-A meta-analysis of imaging studies on the self. NeuroImage, 31, 440-457.

OCHSNER, K. N. (2000). Are affective events richly recollected or simply familiar? The experience and process of recognizing feelings past. Journal of Experimental Psychology: General, 129, 242-261.

Otten, L. J., \& RugG, M. D. (2001). When more means less: Neural activity related to unsuccessful memory encoding. Current Biology, 11, 1528-1530.

Packard, M. G., Cahill, L., \& McGaugh, J. L. (1994). Amygdala modulation of hippocampal-dependent and caudate nucleus-dependent memory processes. Proceedings of the National Academy of Sciences, 91, 8477-8481.
Paller, K. A., \& Wagner, A. D. (2002). Observing the transformation of experience into memory. Trends in Cognitive Sciences, 6, 93-102.

Petrides, M. (2007). The orbitofrontal cortex: Novelty, deviation from expectation, and memory. In G. Schoenbaum, J. A. Gottfried, E. A. Murray, \& S. J. Ramus (Eds.), Linking affect to action: Critical contributions of the orbitofrontal cortex (Annals of the New York Academy of Sciences, Vol. 1121, pp. 33-53). New York: New York Academy of Sciences.

Phelps, E. A. (2006). Emotion and cognition: Insights from studies of the human amygdala. Annual Review of Psychology, 57, 27-53.

Ranganath, C., Yonelinas, A. P., Cohen, M. X., Dy, C., Tom, S. M., \& D'Esposito, M. (2003). Dissociable correlates of recollection and familiarity within the medial temporal lobes. Neuropsychologia, 42, 2-13.

Reisberg, D., \& Hertel, P. (Eds.) (2004). Memory and emotion. New York: Oxford University Press.

Richardson, M. P., Strange, B. A., \& Dolan, R. J. (2004). Encoding of emotional memories depends on amygdala and hippocampus and their interactions. Nature Neuroscience, 7, 278-285.

Rowe, G., Hirsh, J. B., \& Anderson, A. K. (2007). Positive affect increases the breadth of attentional selection. Proceedings of the $\mathrm{Na}$ tional Academy of Sciences, 104, 383-388.

Sharot, T., Delgado, M. R., \& Phelps, E. A. (2004). How emotion enhances the feeling of remembering. Nature Neuroscience, 7 , 1376-1380.

SLotnick, S. D., \& Dodson, C. S. (2005). Support for a continuous (single-process) model of recognition memory and source memory. Memory \& Cognition, 33, 151-170.

Slotnick, S. D., Moo, L. R., Segal, J. B., \& Hart, J., JR. (2003). Distinct prefrontal cortex activity associated with item memory and source memory for visual shapes. Cognitive Brain Research, 17, 75-82.

TAlairach, J., \& Tournoux, P. (1988). Co-planar stereotaxic atlas of the human brain: 3-dimensional proportional system: An approach to cerebral imaging (M. Rayport, Trans.). New York: Thieme.

Van Overschelde, J. P., Rawson, K., \& Dunlosky, J. (2004). Category norms: An updated and expanded version of the Battig and Montague (1969) norms. Journal of Memory \& Language, 50, 289-335.

WAGNer, A. D., \& DaVACHI, L. (2001). Cognitive neuroscience: Forgetting of things past. Current Biology, 11, R964-R967.

Wagner, A. D., Paré-Blagoev, E. J., Clark, J., \& Poldrack, R. A. (2001). Recovering meaning: Left prefrontal cortex guides controlled semantic retrieval. Neuron, 31, 329-338.

Wais, P. E., Wixted, J. T., Hopkins, R. O., \& Squire, L. R. (2006). The hippocampus supports both the recollection and the familiarity components of recognition memory. Neuron, 49, 459-466.

WheELER, M. E., \& BUCKNER, R. L. (2003). Functional dissociation among components of remembering: Control, perceived oldness, and content. Journal of Neuroscience, 23, 3869-3880.

YonELinAs, A. P. (2002). The nature of recollection and familiarity: A review of 30 years of research. Journal of Memory \& Language, 46, 441-517.

\section{NOTES}

1. The regions identified by this technique were also identified in interaction analyses (e.g., examining regions that were more strongly related to negative remembered vs. known than to positive remembered vs. known). However, the benefit to the masking technique is that it reveals only the regions that are significantly active in the contrast of interest. By contrast, significant interactions can emerge due to trends in opposite directions (i.e., even when the activity is not significant in the contrast of interest). For this reason, we report those results identified through the masking technique. We then confirmed, using an ANOVA, that the regions revealed through this technique did indeed show a significant interaction between valence and subsequent response type.

2. One might imagine that this visual activity at encoding may be driven by the processing of the pictures. Indeed, when the data were analyzed separately for words and pictures, this was the case.

(Manuscript received August 30, 2007; revision accepted for publication December 30, 2007.) 